\title{
Composition of Change in Phase Angle with Age in Japanese Children
}

\author{
Katsunori Fujii ${ }^{1, ~ *}$, Kentaro Hayakawa ${ }^{2}$ \\ ${ }^{1}$ Graduate School of Business Administration and Computer Science, Aichi Institute of Technology, Toyota City, Aichi, Japan \\ ${ }^{2}$ Child Studies, Nagoya Management Junior College, Owariasahi City, Aichi, Japan
}

Email address:

fujii@aitech.ac.jp (K. Fujii), k-hayakawa@nagoya-su.ac.jp (K. Hayakawa)

${ }^{*}$ Corresponding author

\section{To cite this article:}

Katsunori Fujii, Kentaro Hayakawa. Composition of Change in Phase Angle with Age in Japanese Children. American Journal of Sports Science. Vol. 8, No. 1, 2020, pp. 10-16. doi: 10.11648/j.ajss.20200801.12

Received: February 17, 2020; Accepted: February 26, 2020; Published: March 10, 2020

\begin{abstract}
Phase angle (PhA) is a value derived from the resistance $(\mathrm{R})$ in intra- and extracellular fluid and the reactance $(\mathrm{Xc})$ in the cell membrane, measured by bioelectrical impedance analysis (BIA). It has also been referred to as a nutritional index that shows the level of cellular activation, becoming a focus of attention in recent years. However, very little is known about the changes in phase angle with age, particularly the changes that occur during the school-age years. In this study, we investigated whether phase angle has a maximum peak velocity (MPV) similar to that of physique, and if a pubertal peak exists whether it is meaningful as a biological parameter in the same way as height growth. The results showed that the age at MPV of phase angle may be proposed as a critical point in the acquisition of health with stronger physical growth and development during the school-age years. Assuming that MPV of phase angle is a critical point in physical growth and development, its involvement in disease needs to be closely observed with this critical point as a juncture since this is also a time period when the thymus of the immune system shows a pubertal peak. At the same time, beyond the pubertal peak of physique, this may be considered a period during which more adult-like physical enhancement and level of health is maintained.
\end{abstract}

Keywords: Phase Angle, Bioelectrical Impedance Analysis (BIA), Maximum Peak Velocity (MPV),

Wavelet Interpolation Model

\section{Introduction}

Many studies have analyzed the risk of obesity with respect to disease, but this is just risk; obesity cannot be called a disease. It is also plainly true that the standard for obesity has been made clear in studies on obesity. For example, standardization of obesity based on BMI. Although BMI standards differ by nationality, race, and ethnicity, people tend to be obese when BMI exceeds 25-30. However, while standardization of obesity with BMI is possible, there is no cut-off value for disease [1]. Bioelectric impedance analysis (BIA), which measures body tissue non-invasively, has also spread and become common in recent years. With BIA it is possible to estimate things such as body fat and muscle mass, and the composition inside the body has become clearer. This means that obesity standards can now be easily grasped from body fat. However, while obesity is still not said to be a disease, it has also become clear that it is not healthy. There is also no guarantee that a person can be called healthy simply because body fat percentage and BMI are in the normal range. Thus, while body fat percentage derived from BMI or BIA is used as a simple measure of fatness or thinness, it is not an indicator of health [2].

There is an index considered to be an indicator of nutritional status that has gained considerable attention in recent years. That index uses the resistance originating in intra- and extracellular fluid and the reactance originating in the cell membrane, measured with BIA, and the vector value of impedance $(Z)$ expressed as the square root of the square of $R$ and the square of Xc. Likening the cell membrane to a condenser, Xc delays current temporally more than voltage, giving rise to a phase shift. The phase angle ( $\mathrm{PhA})$ is the arc tangent of Xc divided by R. Consequently, the phase angle shows the relationship between the status of the cell membrane and the intracellular fluid. The phase angle shows 
lower values when the number of dead cells increases or when cell permeability decreases, and higher values when there are many healthy cells $[3,4]$. It has been reported to be effective in predicting the survival rate of cancer patients [5]. Tsutsumi et al. found a significant correlation between disease severity and phase angle in patients with serious illness, but stated that no certain conclusions have been reached about whether it is an effective indicator of outcome [6]. However, the possibility has been shown that it has a high level of usefulness as a nutritional indicator.

Phase angle is also known as a muscle quality parameter since it reflects the relationship of intra- and extracellular fluid calculated from $\mathrm{R}$ and $\mathrm{Xc}[7,8]$. Phase angle is considered to increase with increases in muscle mass or intracellular fluid in muscle $[9,10]$. Uemura et al. pointed out that in addition to being useful in assessments of frailty and sarcopenia in elderly people and as an indicator of disease outcome, it acts as a general indicator showing the changes in physical health with age [11]. Of course, phase angle differs by race, and is reported to be higher in Caucasoid than in Mongoloid people. The age-related changes in phase angle have been shown with data from Americans. According to those findings, phase angle tends to decrease with age past adulthood. However, the age-related changes in phase angle in Japanese people have not been reported.

There is very little information on the meaning of age-related changes in phase angle. Barbosa-Silva et al. showed that in Americans, phase angle values are low until adulthood, reach a peak value at age 20-30, and afterward a decline [12]. Thus, phase angle reaches a peak in the adult body, and in subsequent age-related changes shows a decrease. This indicates that the level of cellular activity decreases together with the decrease in muscle mass. That phase angle shows low values in school-age children is thought to be because muscle mass has not reached the adult level, as the body is still growing and developing. However, there are not necessarily any clear findings related to such information. In this study, therefore, we focused on Japanese schoolchildren and analyzed the age-related changes in phase angle to answer the questions of whether there is a sudden increase during puberty as in physique, and if there is such a pubertal peak, whether it is meaningful as a biological parameter such as growth in height.

\section{Methods}

\subsection{Subjects and Materials}

Body composition was measured annually from 2015 to 2018 in children aged 6 to 15 years old at elementary and junior high schools in one prefecture of Japan, and data were obtained for 5,342 boys and 5,098 girls. The details are shown in Table 1. The subjects' birthdays were also surveyed and their exact ages on the days of measurement were calculated. The survey and measurements were explained in advance to the subjects and their parents, and informed consent was obtained. None of the subjects had any acute or chronic diseases.
Table 1. Number of males and Females aged 6 to 15.

\begin{tabular}{lll}
\hline \multirow{2}{*}{ Age } & Boys & Girls \\
\cline { 2 - 3 } & (n) & (n) \\
\hline 6 & 350 & 303 \\
7 & 689 & 680 \\
8 & 669 & 699 \\
9 & 737 & 700 \\
10 & 791 & 717 \\
11 & 856 & 758 \\
12 & 529 & 494 \\
13 & 265 & 303 \\
14 & 301 & 290 \\
15 & 155 & 154 \\
\hline
\end{tabular}

\subsection{Phase Angle Measurement}

Using BIA with a TANITA MC-780 body composition analyzer, height, weight, body fat percentage, fat mass, muscle mass, and estimated bone mass were measured. Resistance (R), reactance $(\mathrm{Xc})$, impedance $(\mathrm{Z})$, and phase angle were also measured.

\subsection{Analytical Procedures}

1) Cross-sectional age-related data on physique and body composition were gathered for each school year from the first year of elementary school until the third year of junior high school, and statistical values were calculated as physical growth data for those years.

2) Normality was tested in each school year for the mean age-related change in phase angle from the first year of elementary school until the third year of junior high school. One-way analysis of variance was performed and if there was a significant difference a multiple comparison was conducted to analyze the trends in age-related change in phase angle.

3) The wavelet interpolation model was applied to the mean age-related change values from the first year of elementary school to the third year of junior high school for height, weight, BMI, body fat percentage, muscle percentage, muscle versus height ratio of muscle mass divided by height, and phase angle.

4) The age at maximum peak velocity (MPV) was identified on the age-related change velocity curve for the elements to which the wavelet interpolation model was applied. A comparison between those elements was then attempted.

\subsection{Wavelet Interpolation Model}

The wavelet interpolation model (WIM) is a method in which a growth distance curve is drawn with interpolation between data with a wavelet function (Meyer's mother wavelet is the base function), the distance curve drawn is differentiated and the obtained growth velocity curve is derived. The growth distance values at the pubertal peak and the age of menarche were investigated in this study to approximately describe the true growth curve from the given growth data. The characteristics of the wavelet interpolation model are that local phenomena are read sensitively and the 
approximation accuracy is very high. The details of the theoretical background and the grounds for its effectiveness have already been described in Fujii's previous research [13], so the data analysis algorithm using the wavelet interpolation model is omitted here. The wavelet interpolation model was applied to the age distance value of Phase angle from 6 to 15 years of age in boys and girls. Then, the age distance curves for Phase angle in boys and girls were differentiated, and the age at MPV of Phase angle (age at peak during puberty) from the velocity curve was specified through this process.

\section{Results}

\subsection{Test of Phase Angle Normality with Age}

Normality was tested from the statistics for phase angle in each school year in boys and girls from the first year of elementary school (6 years old) to the third year of junior high school (14, 15 years old) (Tables 2, 3). There are ways to investigate kurtosis and skewness from the distribution of data in tests of normality, but in this study the analysis was done with the application of a normal distribution function from the frequency distribution of the actual phase angle.

The results are shown in Tables 4,5 . The results of a $\chi^{2}$ test between the theoretical values, derived from the normal distribution function of phase angle, and the actual values in boys and girls of all grades showed normality with no significant differences. Figures 1 and 2 show the phase angle frequency distributions in boys and girls in the first year of elementary school. These distributions are seen to fit a normal distribution function well.

Table 2. Statistics of height, weight, BMI and Phase angle in boys.

\begin{tabular}{lllll}
\hline Age & Height $(\mathbf{c m})$ & Weight $(\mathbf{k g})$ & BMI $\left(\mathbf{k g} / \mathbf{m}^{\mathbf{2}}\right)$ \\
\hline 6 & $117.45 \pm 5.35$ & $22.14 \pm 4.37$ & $15.94 \pm 2.1$ & Phase Angle \\
7 & $122.33 \pm 5.26$ & $24.45 \pm 4.84$ & $16.23 \pm 2.28$ & $5.11 \pm 0.40$ \\
8 & $127.69 \pm 5.5$ & $27.76 \pm 6.37$ & $16.90 \pm 3.00$ & $5.13 \pm 0.41$ \\
9 & $133.33 \pm 5.55$ & $31.32 \pm 7.60$ & $17.48 \pm 3.32$ & $5.23 \pm .042$ \\
10 & $138.71 \pm 6.22$ & $35.48 \pm 9.18$ & $18.26 \pm 3.60$ & $5.28 \pm 0.43$ \\
11 & $145.18 \pm 7.29$ & $39.79 \pm 10.19$ & $18.69 \pm 3.56$ & $5.32 \pm 0.44$ \\
12 & $151.54 \pm 7.29$ & $43.4 \pm 10.02$ & $18.75 \pm 3.27$ & $5.34 \pm 0.45$ \\
13 & $159.69 \pm 7.81$ & $50.21 \pm 10.92$ & $20.34 \pm 3.93$ & $5.69 \pm 0.47$ \\
14 & $164.89 \pm 6.92$ & $55.50 \pm 12.06$ & $20.30 \pm 3.51$ \\
15 & $167.72 \pm 5.82$ & $57.24 \pm 11.05$ & $5.95 \pm 0.57$ \\
\hline
\end{tabular}

Table 3. Statistics of height, weight, BMI and Phase angle in girls.

\begin{tabular}{lllll}
\hline Age & Height $(\mathbf{c m})$ & Weight $(\mathbf{k g})$ & BMI $\left(\mathbf{k g} / \mathbf{m}^{\mathbf{2}}\right)$ \\
\hline 6 & $116.76 \pm 4.88$ & $21.48 \pm 3.92$ & $15.66 \pm 2.00$ & Phase Angle \\
7 & $121.12 \pm 5.32$ & $23.54 \pm 4.43$ & $15.96 \pm 2.22$ & $4.98 \pm 0.37$ \\
8 & $126.81 \pm 5.63$ & $26.51 \pm 5.29$ & $16.39 \pm 2.46$ & $5.03 \pm 0.38$ \\
9 & $132.93 \pm 6.24$ & $30.08 \pm 6.52$ & $16.91 \pm 2.81$ & $5.07 \pm 0.39$ \\
10 & $139.66 \pm 6.81$ & $34.57 \pm 7.85$ & $17.58 \pm 3.03$ & $5.06 \pm 0.39$ \\
11 & $146.67 \pm 6.66$ & $39.31 \pm 8.71$ & $19.15 \pm 3.15$ & $5.07 \pm 0.39$ \\
12 & $151.79 \pm 5.70$ & $44.35 \pm 9.42$ & $19.89 \pm 2.96$ & $5.18 \pm 0.44$ \\
13 & $154.47 \pm 5.48$ & $47.59 \pm 8.27$ & $20.49 \pm 2.96$ \\
14 & $156.63 \pm 5.38$ & $50.37 \pm 8.25$ & $21.00 \pm 2.51$ \\
15 & $156.79 \pm 4.83$ & $51.65 \pm 6.84$ & $5.38 \pm 0.52$ \\
\hline
\end{tabular}

Table 4. Test of normality of Phase Angle value from 6 to 15 years.

\begin{tabular}{lll}
\hline & Boys & Girls \\
\hline 6 years old & $f(x)=\frac{0.2 \times 350}{\sqrt{2 \pi} \times 0.4} e^{-\frac{\left(x_{i}-5.11\right)^{2}}{2(0.4)^{2}}}$ & $f(x)=\frac{0.2 \times 303}{\sqrt{2 \pi} \times 0.37} e^{-\frac{\left(x_{i}-4.98\right)^{2}}{2(0.37)^{2}}}$ \\
7 years old & $f(x)=\frac{0.2 \times 689}{\sqrt{2 \pi} \times 0.41} e^{-\frac{\left(x_{i}-5.13\right)^{2}}{2(0.41)^{2}}}$ & $f(x)=\frac{0.2 \times 680}{\sqrt{2 \pi} \times 0.38} e^{-\frac{\left(x_{i}-5.03\right)^{2}}{2(0.38)^{2}}}$ \\
9 years old & $f(x)=\frac{0.2 \times 669}{\sqrt{2 \pi} \times 0.42} e^{-\frac{\left(x_{i}-5.23\right)^{2}}{2(0.42)^{2}}}$ & $f(x)=\frac{0.2 \times 699}{\sqrt{2 \pi} \times 0.39} e^{-\frac{\left(x_{i}-5.07\right)^{2}}{2(0.39)^{2}}}$ \\
10 years old & $f(x)=\frac{0.2 \times 737}{\sqrt{2 \pi} \times 0.43} e^{-\frac{\left(x_{i}-5.28\right)^{2}}{2(0.43)^{2}}}$ & $f(x)=\frac{0.2 \times 700}{\sqrt{2 \pi} \times 0.39} e^{-\frac{\left(x_{i}-5.06\right)^{2}}{2(0.39)^{2}}}$ \\
11 years old & $f(x)=\frac{0.2 \times 791}{\sqrt{2 \pi} \times 0.44} e^{-\frac{\left(x_{i}-5.32\right)^{2}}{2(0.44)^{2}}}$ & $f(x)=\frac{0.2 \times 717}{\sqrt{2 \pi} \times 0.39} e^{-\frac{\left(x_{i}-5.07\right)^{2}}{2(0.39)^{2}}}$ \\
12 years old & $f(x)=\frac{0.2 \times 856}{\sqrt{2 \pi} \times 0.45} e^{-\frac{\left(x_{i}-5.34\right)^{2}}{2(0.45)^{2}}}$ & $f(x)=\frac{0.2 \times 758}{\sqrt{2 \pi} \times 0.41} e^{-\frac{\left(x_{i}-5.06\right)^{2}}{2(0.41)^{2}}}$ \\
13 years old & $f(x)=\frac{0.2 \times 529}{\sqrt{2 \pi} \times 0.47} e^{-\frac{\left(x_{i}-5.40\right)^{2}}{2(0.47)^{2}}}$ & $f(x)=\frac{0.2 \times 494}{\sqrt{2 \pi} \times 0.44} e^{-\frac{\left(x_{i}-5.18\right)^{2}}{2(0.44)^{2}}}$ \\
14 years old & $f(x)=\frac{0.2 \times 265}{\sqrt{2 \pi} \times 0.47} e^{-\frac{\left(x_{i}-5.69\right)^{2}}{2(0.47)^{2}}}$ & $f(x)=\frac{0.2 \times 303}{\sqrt{2 \pi} \times 0.52} e^{-\frac{\left(x_{i}-5.38\right)^{2}}{2(0.52)^{2}}}$ \\
15 years old & $f(x)=\frac{0.2 \times 301}{\sqrt{2 \pi} \times 0.57} e^{-\frac{\left(x_{i}-5.95\right)^{2}}{2(0.57)^{2}}}$ & $f(x)=\frac{0.2 \times 290}{\sqrt{2 \pi} \times 0.49} e^{-\frac{\left(x_{i}-5.45\right)^{2}}{2(0.49)^{2}}}$ \\
\hline
\end{tabular}


Table 5. $\chi^{2}$ value for boys and girls from 6 to 15 years.

\begin{tabular}{lll}
\hline Age & Boys & Girls \\
\hline 6 & 15.16 & 4.55 \\
7 & 15.78 & 12.81 \\
8 & 9.14 & 18.91 \\
9 & 18.62 & 8.31 \\
10 & 27.00 & 12.91 \\
11 & 21.97 & 6.25 \\
12 & 18.26 & 11.40 \\
13 & 10.10 & 142.19 \\
14 & 16.91 & 38.22 \\
15 & 11.87 & 19.35 \\
\hline
\end{tabular}

\subsection{Trends in the Changes with Age in Height, Weight and BMI}

The trends in the changes with age in height, weight, and BMI described by the wavelet interpolation model are shown in Figures 3-8. The respective ages at MPV determined from these graphs are shown in Table 6. In boys, the age at MPV is 13.2 years for height, 13.4 years for weight, and 13.5 years for BMI. In girls, these ages are 10.5 years for height, 11.8 years for weight, and 12.2 years for BMI. Although these are MPV ages derived from mean cross-sectional data, they are thought to be standard results. Thus, the order in which age at MPV for height, weight, and BMI appears follows that in previous studies $[1,14]$.

Table 6. Statistics of age at MPV and MPV in each item.

\begin{tabular}{lllll}
\hline & \multicolumn{3}{l}{ Boys } & Girls \\
\cline { 2 - 5 } & Age at MPV & MPV & Age at MPV & MPV \\
\hline Height & 13.20 & 8.68 & 10.5 & 7.19 \\
Weight & 13.40 & 2.90 & 11.8 & 5.25 \\
BMI & 13.50 & 1.16 & 12.2 & 1.09 \\
Phase angle & 13.5 & 0.36 & 12.8 & 0.18 \\
\hline
\end{tabular}

\subsection{Analysis of Trends in the Change with Age in Phase Angle and the Age at MPV}

Analyses of variance were conducted for the changes with age in phase angle in each school year for boys and girls from 6 to 15 years old. Multiple comparisons were conducted for each school year from age 6 from the relationships found to have significant differences in these analyses of variance. As shown in Tables 2, 3, higher values were shown for higher grades for all school years from 6 years old to 8,9 , and 11 years old and junior high school.

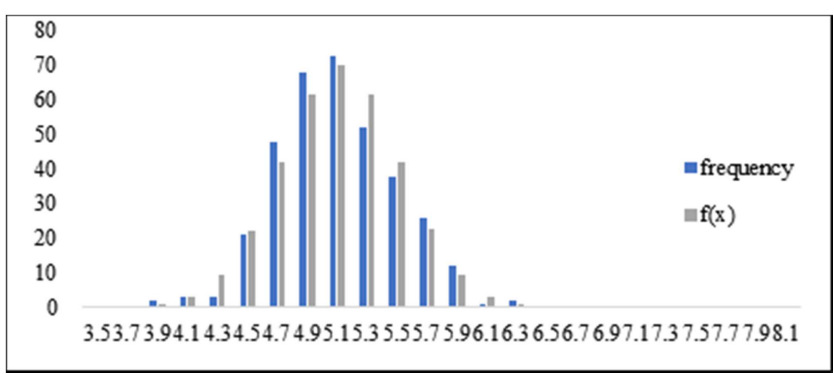

Figure 1. Frequency distribution of phase angle in 6 year old boys.

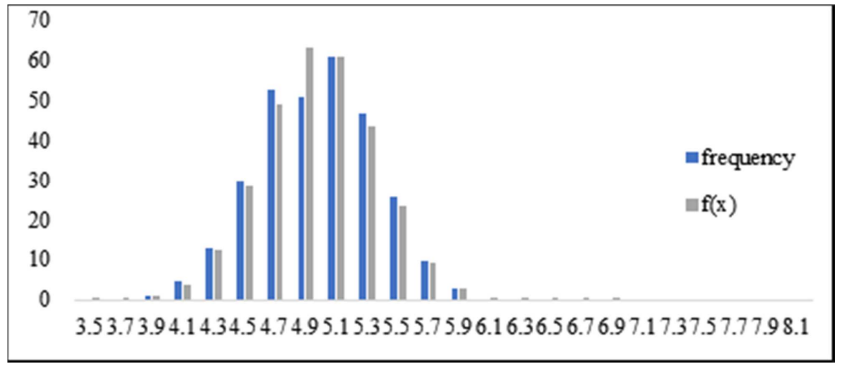

Figure 2. Frequency distribution of phase angle in 6 year old girls.

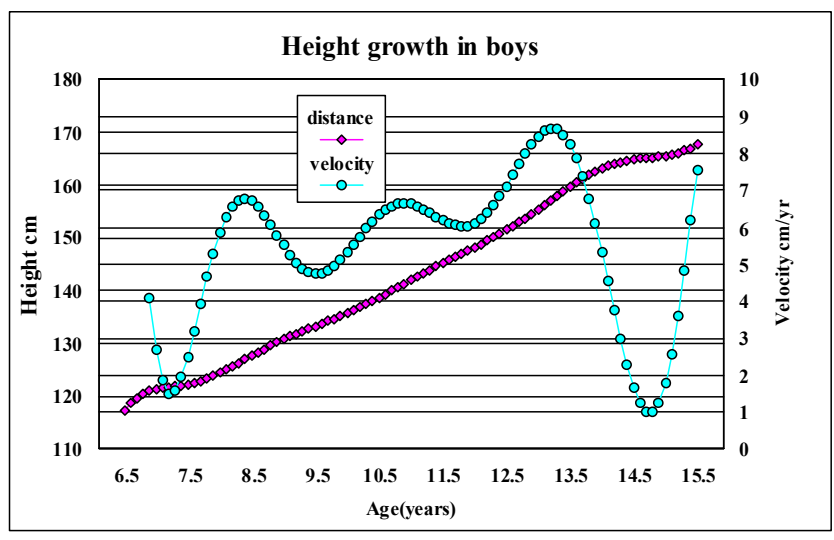

Figure 3. Height growth and the age at MPV derived from wavelet interpolation model in boys.

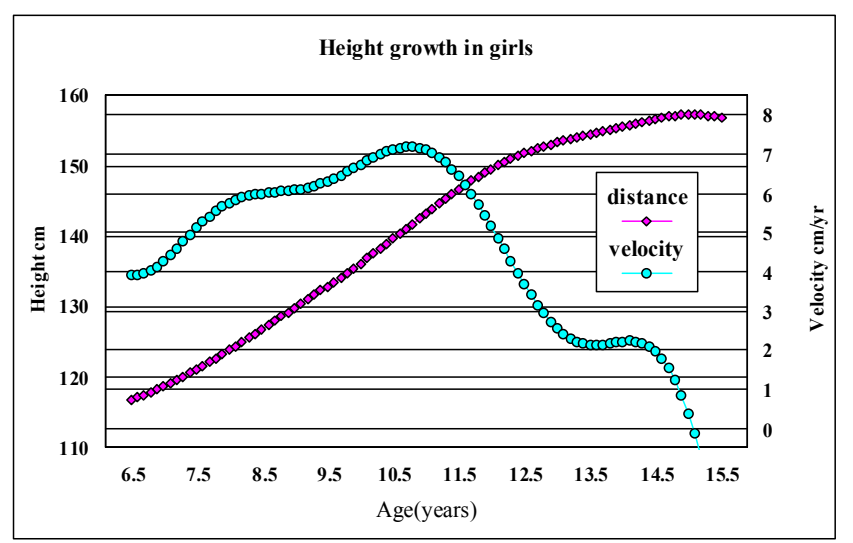

Figure 4. Height growth and the age at MPV derived from wavelet interpolation model in girls.

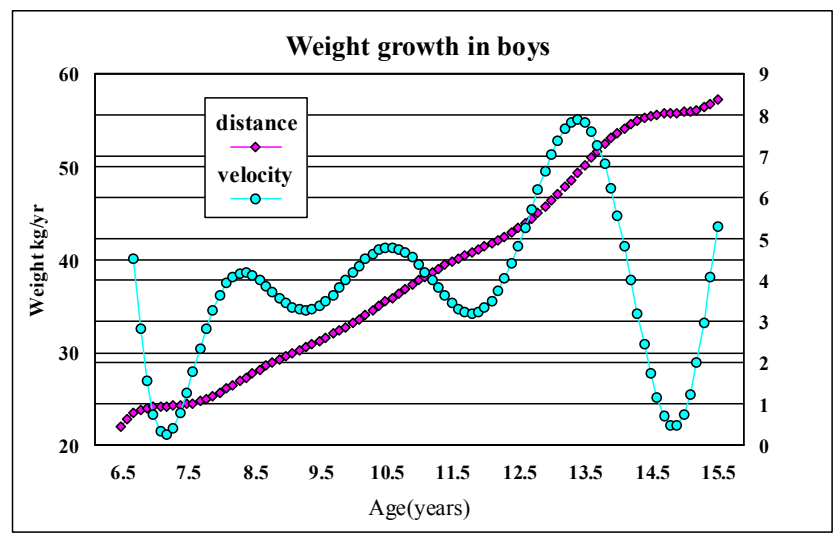

Figure 5. Weight growth and the age at MPV derived from wavelet interpolation model in boys. 


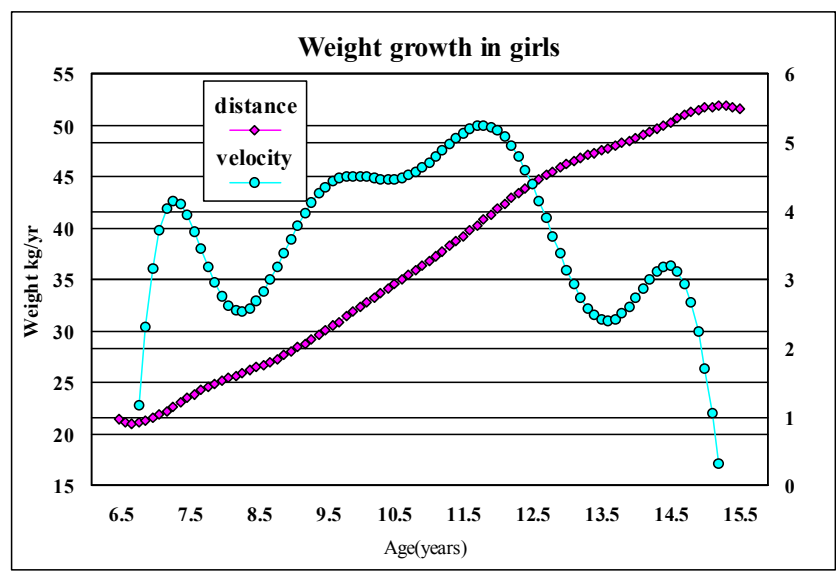

Figure 6. Weight growth and the age at MPV derived from wavelet interpolation model in girls.

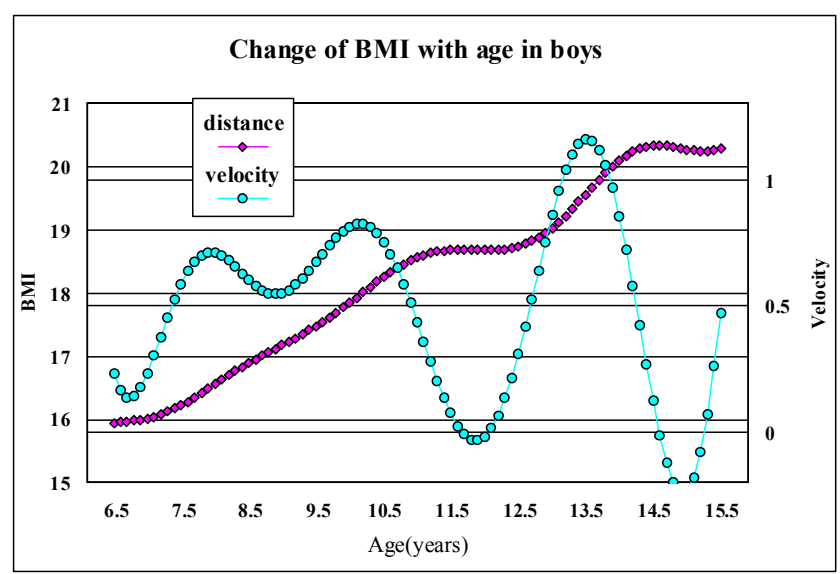

Figure 7. Change of BMI with age and the age at MPV derived from wavelet interpolation model in boys.

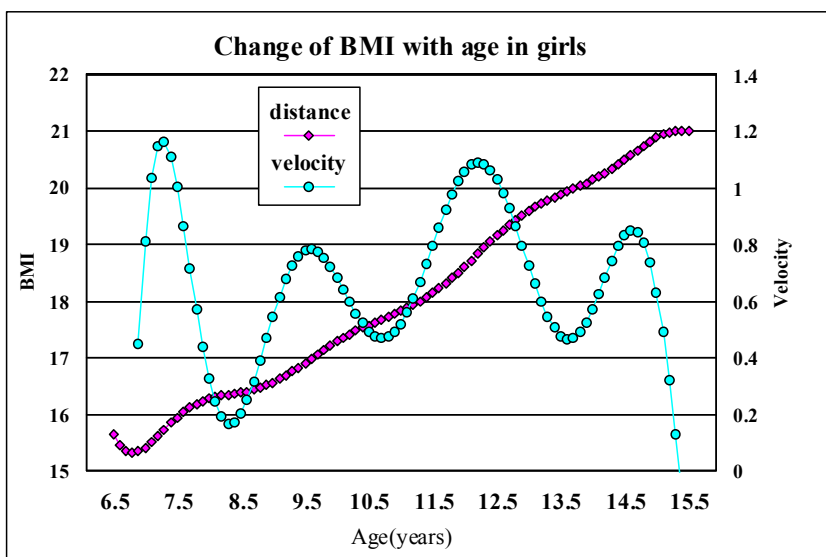

Figure 8. Change of BMI with age and the age at MPV derived from wavelet interpolation model in girls.

The wavelet interpolation model was then applied to the mean values for phase angle in each school year in boys and girls. Figures 9 and 10 show phase angle aging distance values and velocity curves for boys and girls described with the wavelet interpolation model. Looking at the phase angle velocity curves for boys and girls, it is seen that there is a prominent, rapid increase during puberty. It is clear that the velocity for boys is larger. The age at MPV for phase angle was shown to be 13.5 years old for boys and 12.8 years old for girls; earlier in girls. These findings are thought to be a sex difference. Comparison with the described MPV age for height, weight, and BMI reveals that the MPV age for phase angle is the oldest in both boys and girls. In other words, the peak for phase angle appears after the peaks for elements of physique.

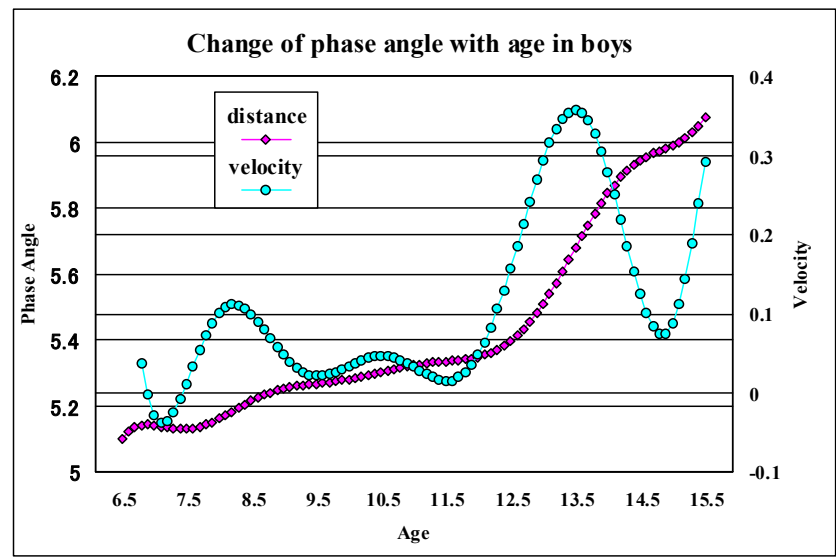

Figure 9. Change of phase angle with age and the age at MPV described from wavelet interpolation model in boys.

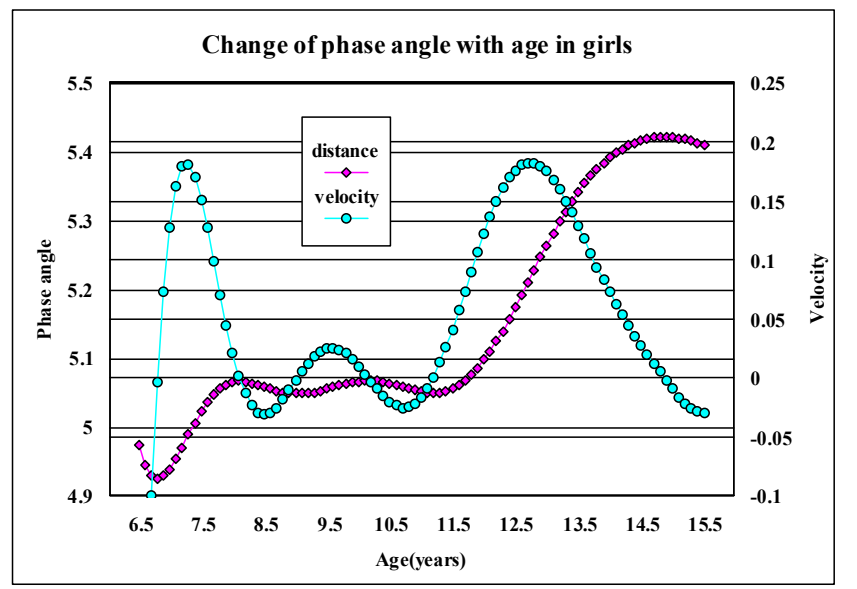

Figure 10. Change of phase angle with age and the age at MPV described from wavelet interpolation model in girls.

\section{Discussion}

In this study, an analysis of the changes with age in phase angle was performed for boys and girls from 6 to 15 years old. There are almost no previous reports on the changes with age in phase angle during the school years. Findings from western countries with age divided into large spans exist $[12,14]$, but they are changes with age from the time adulthood is reached until middle age, and phase angle is reported to peak in adulthood and decrease in middle age. There are no previous findings showing the changes with age in puberty as shown in this study. It may also be said that the results showing normality against the frequency distribution of phase angle in all school years are a new finding of this study. Phase angle, like BMI, is thought to be an element that does not show normality, but when phase angle is judged in limited spans it is 
probably important to conduct investigations with statistical methods that assume normality. Fujii [13] has pointed out that since normality is also seen for BMI during the school years, if one assumes that phase angle is standardized, evaluations that consider standard deviation are probably more meaningful even if a percentile method is not used.

The ability to identify the MPV age for phase angle in this study is quite significant, in that it was possible to compare the age-related velocity curves for height, weight, and BMI, and in the completely new finding that the order of MPV ages could be analyzed. From that order, we see that the MPV age for phase angle appears at the oldest age in both boys and girls. In girls, the MPV age for height is the earliest, followed in order by weight and BMI. The MPV age for phase angle comes after that. However, while the same order is followed for phase angle in boys, the fact that the age is similar to that for MPV of BMI differs from girls. Fujii and Kawanami [15] have pointed out that in the relationship between MPV ages for height and weight, the MPV age for height is on average earlier than that for weight. For girls, that difference is larger than it is for boys. Fujii et al. [1] and Fujii [13] have also pointed out that due to the increase in fat in girls during puberty, the MPV for weight and BMI is larger than that for height, and the difference is bigger than it is in boys. Needless to say, the MPV age for phase angle, like that for height, weight, and BMI, is earlier in girls. This is a sex difference, but that difference is the smallest in the MPV age for phase angle.

Gupta et al. [5] and Tsutsumi et al. [6] have described a relationship between disease, particularly cancer, and phase angle, while Uemura et al. [11] have pointed out that phase angle is an indicator of the activation level of cells. Research on phase angle is still in the very early stage, but phase angle has attracted attention as a simple indicator of health in terms of cell activation and as a nutritional index. If phase angle it taken as an indicator of health, it may be possible to evaluate health levels derived from phase angle by showing the trends in age-related changes in phase angle during the school years. Hence, it may be possible to propose a cutoff value for some types of disease during the school years. In this regard, constructing a standard curve of age-related phase angle is an issue that will likely need to be addressed. However, being able to identify the MPV age for phase angle, in which a pubertal peak appears at a time similar to that for height and weight, may not be that meaningful as an indicator of maturity, like MPV of height. Still, the MPV age for phase angle is the time when cell activation is at its liveliest, and is perhaps a time that facilitates activation of health levels.

Considered in this way, since the MPV ages for BMI and phase angle are nearly the same in boys, the age from 13.5 years onward is potentially a standard for the acquisition of a physically developed, healthy body. Since girls fundamentally mature earlier than boys, a standard for physically developed health is set at the age of 12.8 years and later, following height (MPV age: 10.5 years), weight (MPV age 11.8 years), and BMI (MPV age: 12.2 years). Given that girls mature earlier than boys, more time may be required after the pubertal peak in girls than in boys until a physically developed, healthy body is acquired. The MPV age for phase angle can then be taken as a critical point for the acquisition of physically developed health during the school years. However, as to the meaning of the critical point of phase angle in the school years, if it is taken as a critical point for physical development, then of course it is a period of time in puberty when the immune system thymus peaks, and so it is necessary to look at how it is related to disease with this critical point as a borderline. At the same time, the period following the pubertal peak for physique is a time when young people become more physically like an adult and level of health is ensured. Therefore, if the MPV of height is taken as a biological parameter, it may be that the MPV of phase angle can be proposed as a critical point in physical growth. However, since the MPV age of phase angle was analyzed from cross-sectional data in this study and further verification awaits analyses using longitudinal data, we will limit our discussion to the above.

\section{Conclusion}

The fact that a normality test revealed normality in the frequency distribution of phase angle in each school year for the age-related changes in phase angle in school-age children in Japan is of considerable significance. Moreover, a curve of age-related changes in phase angle was described with the wavelet interpolation model, and identification of the MPV age derived from that velocity curve could be considered a finding derived for the first time in this study. In boys, the MPV ages for BMI and phase angle are nearly the same, and beyond the age of 13.5 years can be considered a standard at which a physically developed, healthy body can be acquired. For girls, a standard for physically developed health is set after the age of 12.8 years, following height, weight, and BMI. In other words, given that girls mature earlier than boys, more time is thought to be needed for girls than for boys until a physically developed, healthy body is acquired. The MPV age for phase angle can be taken as a critical point for the acquisition of physically developed health during the school years. If it is a critical point for physical enhancement, it is also a period of time when the immune system thymus shows a peak in puberty. Thus, it is necessary to look at the relationship with disease taking this critical point as a borderline. At the same time, it may be a period when, more that the pubertal peak for physique, physical development and a health level closer to that of adults is ensured. It may be that the MPV of phase angle can be proposed at a critical point in physical growth.

\section{References}

[1] K. Fujii, T. Ishigaki, M. Sho and Y. Saito, "Health science for life-span development - scientific search to health over life-span -", KyorinShoin, Tokyo, 2008. 
[2] T. Sakai and K. Fujii, "An Approach to Body Composition Study Based on BIA (Bioelectrical Impedance Analysis) Method During Preschool Children", The Journal of Education and Health Science, 50 (3), pp. 177-192, 2014.

[3] Garlini, L. M., Alves, F. D., Ceretta, L. B., Perry, I. S., Souza, G. C., \& Clausell, N. O. "Phase angle and mortality: a systematic review," European Journal of Clinical Nutrition, 73 (4), pp. 495-508, 2019.

[4] Więch, P., Sałacińska, I., Bazaliński, D., \& Dąbrowski, M. "Body composition and phase angle as an indicator of nutritional status in children with juvenile idiopathic arthritis", pediatric Rheumatology, 16 (82), pp. 1-7, 2018.

[5] D. Gupta, C. A. Lammersfeld, P. G. Vashi, "Bioelectrical impedance phase angle in clinical practice - implications for prognosis in stage IIIB and IV non-small cell lung cancer", BMC Cancer, pp. 1-6, 2009.

[6] R. Tsutsumi, J. Oto, K. Fukunaga. et al, "Usefulness and limitation of body composition assessment in critical ill patients", Journal of Japanese Society for Parenteral and Enteral Nutrition, 31 (3), pp. 803-806, 2016.

[7] Sardinha, L. B. "Physiology of exercise and phase angle: another look at BIA", Eur. J. Clin. Nutr, 72, pp. 1323-1327, 2018.

[8] Rodríguez-Rodríguez, F., Cristi-Montero, C., González-Ruíz, K., Correa-Bautista, J. E., \& Ramírez-Vélez, R. "Bioelectrical impedance vector analysis and muscular fitness in healthy men" Nutrients, 8 (407), pp. 1-9, 2016.

[9] Y. Yamada, B. Buehring et al, "Electrical Properties Assessed by Bioelectrical Impedance Spectroscopy as Biomarkers of Age-related Loss of Skeletal Muscle Quantity and Quality", J Gerontol A Biol Sci Med Sci, 72 (9), pp. 1180-1186, 2017.

[10] Langer, R. D., Silva, A. M., Borges, J. H., Cirolini, V. X., Páscoa, M. A., Guerra-Júnior, G., \& Gonçalves, E. M. "Physical training over 6 months is associated with improved changes in phase angle, body composition, and blood glucose in healthy young males", American Journal of Human Biology, 31 (5), pp. 1-8, 2019.

[11] K. Uemura, M. Yamada, K. Saho and H. Okamoto, "Association of Bio-impedance Phase Angle and Physical Activity Level in Older Adults", Journal of Physical Therapy Research, 46 (3), pp. 143-151, 2019.

[12] M C. G. Barbosa-Silva, A JD. Barros, J. Wang, S. B. Heymsfield and R. N. Pierson Jr, "Bioelectrical impedance analysis: population reference values for phase angle by age and sex", American Society for Clinical Nutrition, 82, pp. 49-52, 2005.

[13] K. Fujii, "A scientific approach to growth and development -Physical information science for growth development \& health-," Sankeisha, pp. 112-173, 2006.

[14] Schmidt, S. C., Bosy-Westphal, A., Niessner, C. \& Woll, A. "Representative body composition percentiles from bioelectrical impedance analyses among children and adolescents. The MoMo study”, Clin. Nutr, 38 (6), pp. 2712-2720, 2018.

[15] Fujii, K. and Kawanami, K."An analysis in regard to relationship between age at MPV of height and weight, and its sex difference", Japanese Journal of School Health, 40, pp. 317-331, 1998. 